\title{
Optimum allocation of centers in fuzzy transportation networks with the largest vitality degree
}

\author{
Vitalii Bozheniuk ${ }^{1}$ Alexander Bozhenyuk ${ }^{1}$ Stanislav Belyakov ${ }^{1}$ \\ ${ }^{1}$ Southern Federal University, Nekrasovsky 44, 347922 Taganrog, Russia
}

\begin{abstract}
In this paper the questions of the definition of the centers optimum allocation in fuzzy transportation networks are observed by the minimax criterion. It is supposed that the information received from GIS is presented like a fuzzy graph. In this case the task of the definition of the centers optimum allocation transforms into the task of the definition of the graph vitality fuzzy set. The method and algorithm of the definition of the graph vitality fuzzy set is considered. The example of finding optimum allocation of centers in GIS for railway stations with the largest vitality degree is considered as well.
\end{abstract}

Keywords: Fuzzy graph, fuzzy directed way, accessible degree, fuzzy transitive closure, reachability degree, strong connection degree, vitality degree, fuzzy set of vitality

\section{Introduction}

The large-scale increasing and versatile introduction of a geographical information system (GIS) is substantially connected with the necessity of the perfection of information systems providing decision-making. GIS is applied practically in all spheres of human activity. Geographical information technologies have reached an unprecedented position now, offering a wide range of very powerful functions such as information retrieval and display, analytical tools, and decision support [1, 2]. Unfortunately, geographical data are often analyzed and communicated through largely non-negligible uncertainty. Uncertainty exists in the whole process from geographical abstraction, data acquisition, and geoprocessing to the use $[3,4,5]$.

One of the tasks solved with GIS is the task of the centers allocation [6]. The search of the optimum placing of hospitals, police stations, fire brigades and many other necessary important enterprises and services on some sites of the considered territory are confined to this task. In some cases the criterion of optimality can consist in the minimization of the journey time (or in the minimization of distances) from the service centre to the most remote service station. In other cases the criterion of optimality consists in the choice of such a place of allocating the centers so that the route from them to any other place of service could be passed best by some criterion of a way. In other words, the problem is the optimization of "the worst variant» [7].
However, very often, the information represented in GIS, happens to be of the approximate value or insufficiently authentic [8]. We consider that a certain railway system has $n$ railway stations. There are $k$ service centres, which may be placed into these railway stations. Each centre serves a station, and also some neighbouring stations with the given degree of service. The centers can fail during the exploitation. It is necessary for the given number of centers to define their best allocation. In other words, it is necessary to define the places of $k$ centers into $n$ railway stations so that the "control" of all the territory (all the railway stations) is carried out with the greatest possible service degree.

\section{Basic concepts and definitions}

It is supposed that the service degree of a region is defined as the minimal value from the service degrees of each area (railway station).

Taking into account that the service degree cannot always have a symmetry property (for example, by specific character and relief of the region) the model of this task is a fuzzy directed graph $\tilde{G}=(X, \tilde{U})$ [9]. Here, set $X=\left\{x_{i}\right\}, \quad i \in I=\{1,2, \ldots, n\} \quad$ is a set of vertices and $\tilde{U}=\left\{<\mu_{U}<x_{i}, x_{j}>/<x_{i}, x_{j}>>\right\},<x_{i}, x_{j}>\in X^{2}$ is a fuzzy set of directed edges with a membership function $\mu_{\mathrm{U}}: X^{2} \rightarrow[0,1]$. Membership function $\mu_{U}<x_{i}, x_{j}>$ of fuzzy graph $\tilde{G}=(X, \tilde{U})$ defines a service degree of area $j$ when the center is placed into area $i$. We assume that the service degree has the property of transitivity, i.e. if the service centre is in the area and serves area $x_{j}$ with degree $\mu_{U}<x_{i}, x_{j}>$, and if the service center is in area $x_{j}$ and serves area $x_{k}$ with degree $\mu_{U}\left\langle x_{j}, x_{k}\right\rangle$ then the service degree of area $x_{k}$ from area $x_{i}$ is $\mu_{U}<x_{i}, x_{j}>\& \mu_{U}<x_{j}, x_{k}>$. Here, \& operation is a minimum operation.

The task of the best allocation of the centers in a fuzzy graph can be reduced to the problem of finding a subset of vertices $Y$, from which all the other vertices of fuzzy graph $X / Y$ reachable with the greatest degree. Considering the questions of the optimum allocation of the service centres we will focus on the concepts of a fuzzy directed way, and the degree of fuzzy graph vitality $[9,10]$.

Fuzzy directed way $\tilde{L}\left(x_{i}, x_{j}\right)$ of graph $\tilde{G}=(X, \tilde{U})$ is a sequence of fuzzy directed edges from vertex $x_{i}$ to vertex $x_{m}$ : 
$\tilde{\mathrm{L}}\left(\mathrm{x}_{\mathrm{i}}, \mathrm{x}_{\mathrm{m}}\right)=<\mu_{\mathrm{U}}<\mathrm{x}_{\mathrm{i}}, \mathrm{x}_{\mathrm{j}}>/<\mathrm{x}_{\mathrm{i}}, \mathrm{x}_{\mathrm{j}}>>,<\mu_{\mathrm{U}}<\mathrm{x}_{\mathrm{j}}, \mathrm{x}_{\mathrm{k}}>/$

$<\mathrm{x}_{\mathrm{j}}, \mathrm{x}_{\mathrm{k}}>>, \ldots,<\mu_{\mathrm{U}}<\mathrm{x}_{1}, \mathrm{x}_{\mathrm{m}}>/<\mathrm{x}_{1}, \mathrm{x}_{\mathrm{m}}>>$.

Conjunctive durability of the way $\mu\left(\tilde{L}\left(x_{i}, x_{j}\right)\right)$ is defined as

$$
\mu\left(\tilde{L}\left(x_{i}, x_{m}\right)\right)=\underset{<x_{\alpha}, x_{\beta}>\in \tilde{L}\left(x_{i}, x_{m}\right)}{\&} \mu_{U}<x_{\alpha}, x_{\beta}>.
$$

Fuzzy directed way $\tilde{L}\left(x_{i}, x_{j}\right)$ is called a simple way between vertices $x_{i}$ and $x_{m}$ if its part is not a way between the same vertices.

Vertex $y$ is called a fuzzy accessible of vertex $x$ in graph $\tilde{G}=(X, \tilde{U})$ if the fuzzy directed way from vertex $x$ to vertex $y$ exists.

The accessible degree of vertex $y$ from vertex $x,(x \neq y)$ is defined by the following expression

$$
\gamma(x, y)=\max _{\alpha}\left(\mu\left(\tilde{L}_{\alpha}(x, y)\right), \quad \alpha=1,2, \ldots, p,\right.
$$

where $p$ is the number of various simple directed ways from vertex $x$ to vertex $y$.

On the basis of the presented definition of a fuzzy accessible vertex we can construct accessible matrix $\mathrm{N}$, containing accessible degrees for each pair of the vertices

$$
N=\left\|\gamma_{i j}\right\|_{n}=\bigcup_{k=0}^{n-1} R^{m}
$$

Here, $\gamma_{i j}=\chi\left(x_{i}, x_{j}\right), x_{i} x_{j} \in X, R^{m}$ is the vertex matrix power $m$ for a graph (matrix $R^{0}$ is an identity matrix).

Let us consider that each vertex $x \in X$ in graph $\widetilde{G}=(X, \widetilde{U})$ is accessible from itself with accessible degree $\gamma(x, x)=1$.

Example 1. For the fuzzy graph presented in Fig.1, vertex $x_{5}$ is a fuzzy accessible vertex from $x_{1}$ with the accessible degree

$$
\begin{aligned}
& \gamma\left(\mathrm{x}_{1}, \mathrm{x}_{5}\right)=\max \{(0,7 \& 0,3) ;(0,6 \& 0,8)\}= \\
& =\max \{0,3 ; 0,6\}=0,6 .
\end{aligned}
$$

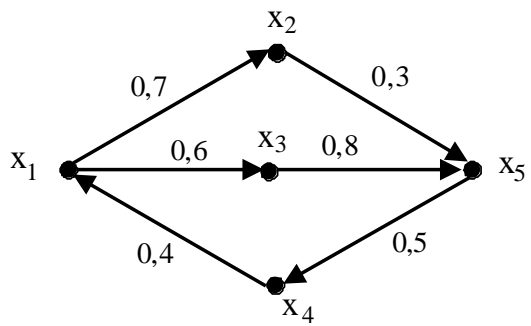

Fig. 1: Fuzzy graph.

Let fuzzy graph $\tilde{G}=(X, \tilde{U})$ be given. The fuzzy multiple-valued reflections $\tilde{\Gamma}^{1}, \tilde{\Gamma}^{2}, \widetilde{\Gamma}^{3}, \ldots, \tilde{\Gamma}^{k}$ are defined as:

$$
\begin{aligned}
& \tilde{\Gamma}^{1}\left(x_{i}\right)=\left\{<\mu_{\Gamma^{1}\left(x_{i}\right)}\left(x_{j}\right) /\left(x_{j}\right)>\right\}, \text { where } \\
& \left(\forall x_{j} \in X\right)\left[\mu_{\Gamma^{1}\left(x_{i}\right)}\left(x_{j}\right)=\mu_{U}<x_{i}, x_{j}>\right], \\
& \tilde{\Gamma}^{2}\left(x_{i}\right)=\tilde{\Gamma}\left\{\tilde{\Gamma}\left(x_{i}\right)\right\}, \tilde{\Gamma}^{3}\left(x_{i}\right)=\tilde{\Gamma}\left\{\tilde{\Gamma}^{2}\left(x_{i}\right)\right\}, \ldots,
\end{aligned}
$$

$$
\begin{aligned}
& \tilde{\Gamma}^{k}\left(x_{i}\right)=\tilde{\Gamma}\left\{\tilde{\Gamma}^{k-1}\left(x_{i}\right)\right\}=\left\{<\mu_{\Gamma^{k}\left(x_{i}\right)}\left(x_{j}\right) / x_{j}>\right\}, \text { where } \\
& \left(\forall x_{j} \in X\right)\left[\mu_{\Gamma^{k}\left(x_{i}\right)}\left(x_{j}\right)=\underset{\forall x_{i} \in X}{\vee} \mu_{\Gamma^{k-1}\left(x_{i}\right)}\left(x_{l}\right) \& \mu_{U}<x_{l}, x_{j}>\right] .
\end{aligned}
$$

It is obvious that $\tilde{\Gamma}^{k}\left(x_{i}\right)$ is a fuzzy subset of the vertices, which are accessible from vertex $x_{i}$, using fuzzy ways with the length $k$.

Example 2. For the fuzzy graph presented in Fig.1 we have

$\tilde{\Gamma}^{1}\left(x_{1}\right)=\left\{<0,7 /\left(x_{2}\right)>,<0,6 / x_{3}>\right\}$,

$\tilde{\Gamma}^{2}\left(x_{1}\right)=\left\{<0,6 / x_{5}>\right\}$.

Fuzzy transitive closure $\hat{\Gamma}\left(x_{i}\right)$ is the fuzzy multiplevalued reflection:

$$
\hat{\Gamma}\left(x_{i}\right)=\tilde{\Gamma}^{0}\left(x_{i}\right) \cup \tilde{\Gamma}\left(x_{i}\right) \cup \tilde{\Gamma}^{2}\left(x_{i}\right) \cup \ldots=\bigcup_{j=0}^{\infty} \tilde{\Gamma}^{j}\left(x_{i}\right) .
$$

Where $\tilde{\Gamma}^{0}\left(x_{i}\right)=\left\{<1 / x_{i}>\right\}$. In other words, vertex $x_{i}$ can reach itself with the degree 1 .

Set $\hat{\Gamma}\left(x_{i}\right)$ is a fuzzy subset of vertices, which are accessible from vertex $x_{i}$ by some fuzzy way with the greatest possible conjunctive degree. Since we consider finite graphs, it is possible to state, that

$$
\hat{\Gamma}\left(x_{i}\right)=\bigcup_{j=0}^{n-1} \tilde{\Gamma}^{j}\left(x_{i}\right) .
$$

Example 3. For the fuzzy graph presented in Fig.1, the fuzzy transitive closure of vertex $x_{1}$ is:

$\hat{\Gamma}\left(x_{1}\right)=\left\{\left\langle 1 / x_{1}\right\rangle,\left\langle 0,7 / x_{2}>,<0,6 / x_{3}\right\rangle,<0,5 / x_{4}>,<0,6 / x_{5}>\right\}$.

Fuzzy graph $\tilde{G}=(X, \tilde{U})$ is a fuzzy strongly connected graph if it satisfies the following condition:

$$
\left(\forall x_{i} \in X\right)\left(S_{\hat{\Gamma}\left(x_{i}\right)}=X\right) .
$$

Here, $S_{\hat{\Gamma}\left(x_{i}\right)}$ is the carrier of fuzzy transitive closure $\hat{\Gamma}\left(x_{i}\right)$.

On the contrary, fuzzy graph $\tilde{G}=(X, \tilde{U})$ is a fuzzy strongly connected graph if there is a fuzzy directed way, the conjunctive strength of which is different from 0 between any two vertices.

Let the fuzzy transitive closure for vertex $x_{i}$ look like $\hat{\Gamma}\left(x_{i}\right)=\left\{<\mu_{i 1}\left(x_{1}\right) / x_{1}>,<\mu_{i 2}\left(x_{2}\right) / x_{2}>, \ldots,<\mu_{i n}\left(x_{n}\right) / x_{n}>\right\}$, then value $\rho(\tilde{G})=\underset{j=1, n}{\&} \underset{i=1, n}{\&} \mu_{i j}\left(x_{j}\right)$ is defined as the degree of the fuzzy graph strong connectivity.

Let $\tilde{G}=(X, \tilde{U})$ be a fuzzy graph with degree of strong connectivity $\rho(\tilde{G})$, and $\tilde{G}^{\prime}=\left(X^{\prime}, \tilde{U}^{\prime}\right)$ be a fuzzy subgraph with degree of strong connectivity $\rho\left(\tilde{G}^{\prime}\right)$.

Fuzzy subgraph $\tilde{G}^{\prime}=\left(X^{\prime}, \tilde{U}^{\prime}\right)$ is defined as a maximum strong connectivity fuzzy subgraph or a fuzzy strong connectivity component if there is no other sub- 
graph $\tilde{G}^{\prime \prime}=\left(X^{\prime \prime}, \tilde{U}^{\prime \prime}\right) \quad$ for $\quad$ which $\quad \tilde{G}^{\prime} \subset \tilde{G}^{\prime \prime}, \quad$ and $\rho\left(\tilde{G}^{\prime}\right) \leq \rho\left(\tilde{G}^{\prime \prime}\right)$.

We consider the degree of fuzzy graph vitality as a degree of strong connection, so it will be defined by the formula $[11,12]$ :

$$
V(\tilde{G})=\underset{x_{i} \in X}{\&} \underset{x_{j} \in X}{\&} \gamma\left(\mathrm{x}_{\mathrm{i}}, \mathrm{x}_{\mathrm{j}}\right) .
$$

It means that there is a route between each pair of the graph vertices with a conjunctive strength not less than value $V$.

To allocate service centers in a fuzzy graph we should consider the problem of the "optimum" placing this way: all (residual) vertices are served with the greatest vitality degree.

Let $k$ be a service center $(k<n)$, placed in the vertices of subset $Y,|Y|=k, Y \subset X$, and $\gamma\left(x_{i}, x_{j}\right)$ is a reachability degree of vertex $x_{j}$ from vertex $x_{i}$.

Definition 1. Value

$$
V_{\tilde{G}}(Y)=\underset{\forall x_{j} \in X \backslash Y}{\&}\left(\underset{\forall x_{i} \in Y}{\vee} \gamma\left(\mathrm{x}_{\mathrm{i}}, \mathrm{x}_{\mathrm{j}}\right) \& \gamma\left(\mathrm{x}_{\mathrm{j}}, \mathrm{x}_{\mathrm{i}}\right)\right)
$$

Is a vitality degree of fuzzy graph $\tilde{\mathrm{G}}$ which is served by $k$-centers from vertex set $Y$.

Vitality degree $\mathrm{v}_{\tilde{\mathrm{G}}}(Y)$ determines the minimax strong connectivity value between each vertex from set $X \backslash Y$ and a center from set $Y$.

In other words, one can "leave" the vertex of subset $Y$, "reach" any vertex of the graph, "serve" it, return to the "initial" vertex while the conjunctive strength of the route will not be less than value $V_{\tilde{G}}(Y)$.

It is clear that value $V_{\tilde{G}}(Y) \in[0,1]$ depends either on the number of centers $k$, or the allocation of the centers on the vertices of graph $\tilde{G}$ (i.e. on the choice of set $Y$ ).

Thus, the problem of the allocation of $k$ service centers $(k<n)$ in fuzzy graph $\tilde{G}$ is reduced to determining such a subset of vertices $Y \subset X$, that value of vitality degree $V_{\tilde{G}}(Y)$ reaches its maximum value, that is value $V_{\tilde{G}}(k)=\max _{\substack{\forall Y \subset X \\|Y|=k}}\left\{V_{\tilde{G}}(Y)\right\} \cdot$

Definition 2. Fuzzy set

$$
\tilde{\mathrm{V}}_{\tilde{\mathrm{G}}}=\left\{<V_{\tilde{G}}(1) / 1>,<V_{\tilde{G}}(2) / 2>, \ldots,<V_{\tilde{G}}(n) / n>\right\},
$$

defined on vertex set $X$, is called a fuzzy set of vitality of graph $\tilde{G}=(X, \tilde{U})$. Fuzzy set of vitality $\tilde{\mathrm{V}}_{\tilde{\mathrm{G}}}$ determines the greatest vitality degrees of graph $\tilde{G}$ if it is served by $1,2 \ldots n$ centers.

Values $\tilde{\mathrm{V}}_{\tilde{\mathrm{G}}}(k)(1 \leq k \leq n)$ signify that we can place $k$ centers in graph $\tilde{G}$ so that there is a route from at least one center to any vertex of graph $\tilde{G}$ and back. The conjunctive strength of the graph will be not less than $\tilde{\mathrm{V}}_{\tilde{\mathrm{G}}}(k)$.

\section{Method for finding centers with the largest vitali- ty degree}

Let us consider the method of finding a family of all service centers with the largest vitality degree. The given method is a similar method for the definition of all fuzzy base sets [13] and fuzzy antibase sets [14] for fuzzy graphs.

Let $Y$ be a subset of the vertices of fuzzy graph $\tilde{G}=(X, \tilde{U})$ in which the service centers are located and the vitality degree equals to $V$. Therefore, one of the two conditions for any vertex $x_{i} \in X$ can be satisfied:

a) vertex $x_{i}$ belongs to set $Y$;

b) there is vertex $x_{j}$ that belongs to set $Y$ and inequalities $\gamma\left(x_{i}, x_{j}\right) \geq V$ and $\gamma\left(x_{j}, x_{i}\right) \geq V$ are encountered.

Using the notation quantifier form we can get the truth of the following formula:

$$
\begin{aligned}
& \left(\forall \mathrm{x}_{\mathrm{i}} \in X\right)\left[x_{i} \in Y \vee\left(\exists x_{j}\right)\right. \\
& \left.\left(x_{j} \in Y \& \gamma\left(x_{i}, x_{j}\right) \geq V \& \gamma\left(x_{j}, x_{i}\right) \geq V\right)\right] .
\end{aligned}
$$

Logical variable $p_{i}$ can be set in correspondence to each vertex $x_{i} \in X$. If $x_{i} \in Y$, the logical variable possesses value 1 , otherwise 0 . Fuzzy variable $\xi_{\mathrm{ij}}=\gamma\left(x_{i}, x_{j}\right)$ can be set in the correspondence to expression $\gamma\left(x_{i}, x_{j}\right) \geq V$. So we can get the truth of the logical formula:

$$
\Phi_{\mathrm{\vee}}=\underset{i=1, n}{\&}\left(p_{i} \vee \underset{j=1, n}{\vee}\left(p_{j} \& \xi_{i j} \& \xi_{i j}\right)\right) .
$$

Let $\xi_{\mathrm{ii}}=1$ and equality

$p_{i} \vee \underset{j}{\vee} p_{i} \& \xi_{i j} \& \xi_{j i}=\underset{j}{\vee} p_{j} \& \xi_{i j} \& \xi_{j i}$ is true for any $x_{i}$ then:

$$
\Phi_{V}=\underset{i=1, n}{\&} \underset{j=1, n}{\vee}\left(\xi_{i j} \& \xi_{j i} \& p_{j}\right) .
$$

While removing the brackets in formula (2), let us use the following rule of absorption:

$$
a v a \& b=a ; \xi^{\prime} \& a v \xi^{\prime \prime} \& a \& b \text {, if } \xi^{\prime} \geq \xi^{\prime} .
$$

Here, $a, b \in\{0,1\}$ and $\xi^{\prime}, \xi^{\prime \prime} \in[0,1]$. Consequently formula (2) will be represented as:

$$
\Phi_{\mathrm{V}}=\underset{i=1, l}{\vee}\left(p_{1_{i}} \& p_{2_{i}} \& \ldots \& p_{k_{i}} \& V_{i}\right) \text {. }
$$

We can prove the following property:

Property. If further simplification in the formula (4) based on the rules (3) is not possible, then the totality of all vertices, conforming to variables, for each disjunctive term $i$ defines the subset of vertices $Y \subseteq X$ with vitality degree $V_{i}$ of fuzzy graph $\tilde{G}=(X, \tilde{U})$. Here subset $Y$ is minimal, in other words, any subset of $Y$ does not have this property.

\section{Algorithm for finding the allocation of service centers}

Let us rewrite expression (2) like this: 


$$
\Phi_{V}=\underset{i=1, n}{\&}\left(a_{i 1} p_{1} \vee a_{i 2} p_{2} \vee \ldots \vee a_{i n} p_{n}\right)
$$

To construct expression (4) we will convert pair $a_{i j} \& p$ from expression (5) to weighted binary vector $a_{i j} \bar{P}_{j}$. Here $\bar{P}_{j}=\left\|p_{i}^{(j)}\right\|$ is a binary vector that has dimension of $n$. The elements of $\bar{P}_{j}$ are defined as:

$$
p_{i}^{(j)}=\left\{\begin{array}{l}
1, \text { if } i=j \\
0, \text { if } i \neq j .
\end{array}\right.
$$

Example 4. Let $\mathrm{n}=3$, so, taking into account our rules of conversion, expression $0.6 \& p_{2}$ is equal to vector $0.6(0,1,0)$ and expression $0.4 \& p_{3}$ is equal to vector $0.4(0,0,1)$.

The conjunction of $\left(a_{1} p_{1}\right)$ and $\left(a_{2} p_{2}\right)$ from expression (5) corresponds the conjunction of two weighted binary vectors $a_{1} \bar{P}_{1} \quad$ and $a_{2} \bar{P}_{2}, \quad \overline{P_{1}}=\left\|p_{i}^{(1)}\right\|, \quad \bar{P}_{2}=\left\|p_{i}^{(2)}\right\|$, $i=\overline{1, n}, a_{1}, a_{2} \in[0,1]$. In a vector space the conjunction is defined as $a_{1} \bar{P}_{1} \& a_{2} \bar{P}_{2}=a \bar{P}$, where $a=\min \left\{a_{1}, a_{2}\right\}$, $\bar{P}=\left\|p_{i}\right\|, p_{i}=\max \left\{p_{i}^{(1)}, p_{i}^{(2)}\right\}, i=\overline{1, n}$.

Example 5. The conjunction of vectors from example (4): $0.6(0,1,0) \& 0.4(0,0,1)=0.4(0,1,1)$.

Let us define the operation $\leq$ "less or equal" between binary vectors. Binary vector $\bar{P}_{1}$ is less or equal than $\bar{P}_{2}$ if and only if each element of $\overline{P_{1}}$ is less or equal than the corresponding element of vector $\bar{P}_{2}$. Or

$$
\left(\bar{P}_{1} \leq \bar{P}_{2}\right) \leftrightarrow(\forall i=\overline{1, n})\left[p_{i}^{(1)} \leq p_{i}^{(2)}\right] .
$$

Example 6. $(0,1,0) \leq(0,1,1)$.

Considering the algebra in space of weighted binary vectors, we can make a rule of absorption:

$$
a_{1} \bar{P}_{1} \vee a_{2} \bar{P}_{2}=a_{1} \bar{P}_{1} \text {, if } a_{1} \geq a_{2} \text { and } \bar{P}_{1} \leq \bar{P}_{2} .
$$

Example 7. $0.6(0,1,0) \vee 0.4(0,1,1)=0.6(0,1,0)$, that corresponds the rule of absorption in space of fuzzy expressions $0.6 \& p_{2} \vee 0.4 \& p_{2} \& p_{3}=0.6 \& p_{2}$.

Now we can construct statement (6) using the conjunction operation and the rule of absorption of weighted binary vectors by the following algorithm:

$1^{\circ}$. Each element of the first bracketed expression $(j=1)$ of expression (5) is converted to weighted binary vector. The result is to be written in the first $n$ elements of the buffer vector $\overline{V_{1}}=\left\|v_{i}^{(1)}\right\|, i=\overline{1, n^{2}}$.

$2^{\circ} . j$ incrementing $(j:=j+1)$.

$3^{\circ}$. Each element of the bracketed expression $j$ is also converted to weighted binary vectors. The result is to be written in the first $n$ elements of the buffer vector $\bar{V}_{2}=\left\|v_{i}^{(2)}\right\|, i=\overline{1, n}$.

$4^{\circ}$. The next stage consists of the conjunction of two vectors $\bar{V}_{1}$ and $\bar{V}_{2}$. The result is placed into the buffer vector $\overline{V_{3}}=\left\|v_{i}^{(3)}\right\|, i=\overline{1, n^{2}}$. While placing elements into $\overline{V_{3}}$, absorption is made using rule (6). $5^{\circ}$. All the elements of buffer $\overline{V_{3}}$ are copied to buffer $\overline{V_{1}}\left(v_{i}^{(1)}:=v_{i}^{(3)}, i=\overline{1, n^{2}}\right)$.

$6^{\circ} . j$ incrementing $(j:=j+1)$.

$7^{\circ}$. If $j \leq n$, the next step is $3^{\circ}$, otherwise $8^{\circ}$.

$8^{\circ}$. Expression (4) is to be built using elements in the buffer $\overline{V_{1}}$. This way we have fuzzy set of vitality $\tilde{V}_{G}$ of graph $\tilde{G}=(X, \tilde{U})$.

\section{Example of the service centers finding}

Let us consider a railway network limited by the stations Novosibirsk, Kemerovo, Barnaul and Novokuzneck. The network is presented in Fig. 2.

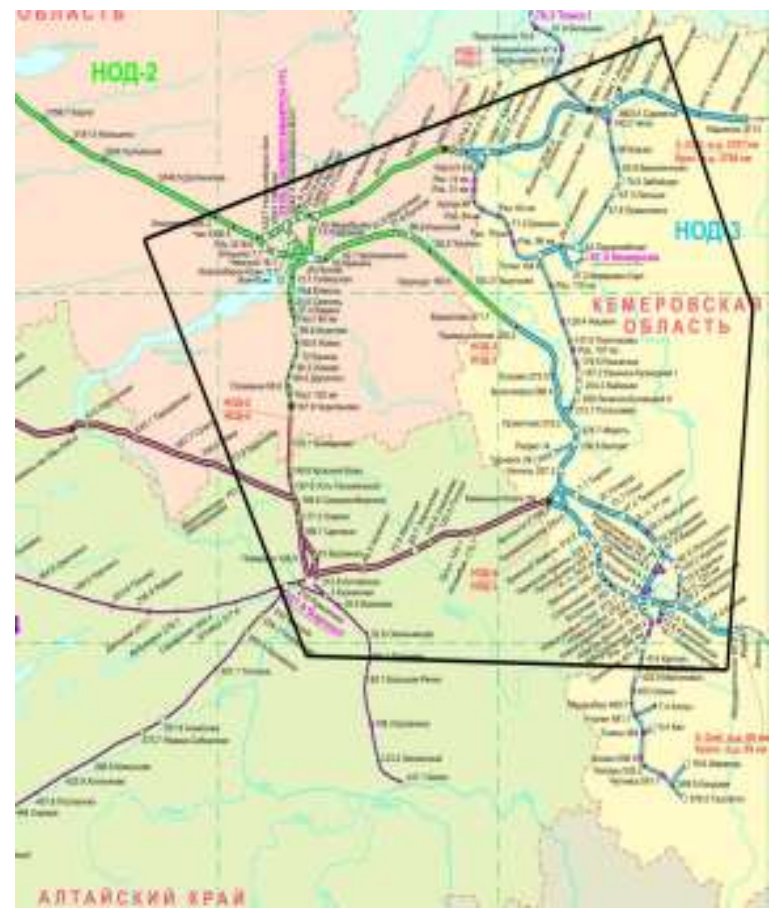

Fig. 2: Railway network.

The fuzzy graph of this railway network, obtained from the GIS "Object Land" [15], is represented in Fig.3. Here the fuzzy graph vertices correspond to the network railway stations, and the fuzzy graph edges correspond to the rail way between the stations. The membership functions of the edges are calculated according to the characteristics of the railway. For example, "if the period of the maintenance of the railway is less than 15 years and the length is less than 20 kilometers, the membership function is equal to 0.9 ". It is necessary to find the allocation of service centers. For the sake of simplicity, let us present all the subgraphs, strong connection degrees of which equals to 1 , by one vertex. 


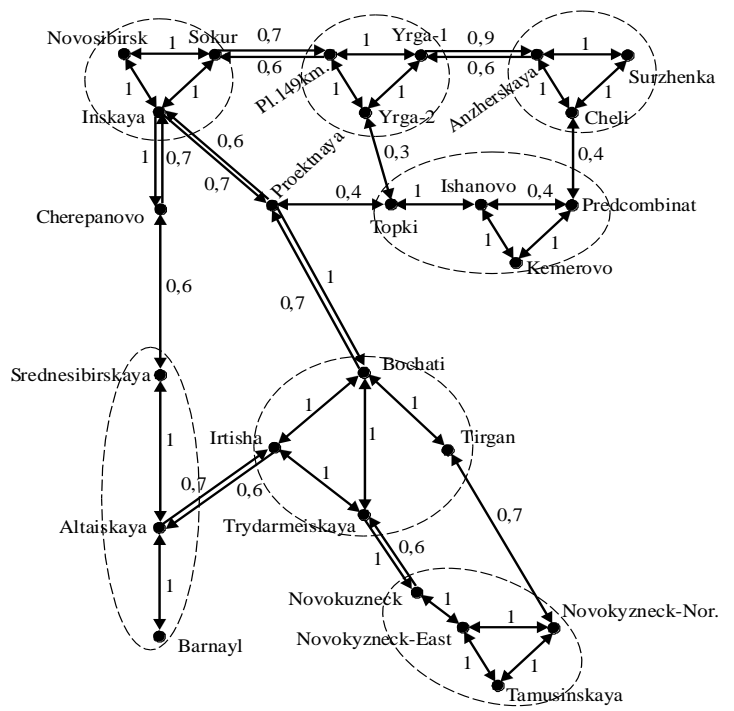

Fig. 3: Fuzzy graph of the railway network.

As a result we will receive the aggregative fuzzy graph with $n=9$, which is represented in Fig. 4:

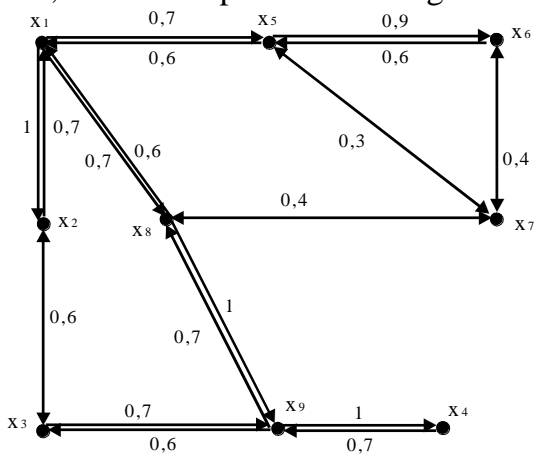

Fig. 4: Aggregative fuzzy graph of the railway network. form

The vertex matrix for this graph has the following

$$
\begin{array}{c|ccccccccc}
x_{1} & x_{2} & x_{3} & x_{4} & x_{5} & x_{6} & x_{7} & x_{8} & x_{9} \\
x_{1} & 0 & 1 & 0 & 0 & 0,7 & 0 & 0 & 0,7 & 0 \\
x_{2} & 0,7 & 0 & 0,6 & 0 & 0 & 0 & 0 & 0 & 0 \\
x_{3} & 0 & 0,6 & 0 & 0 & 0 & 0 & 0 & 0 & 0,7 \\
x_{4} & 0 & 0 & 0 & 0 & 0 & 0 & 0 & 0 & 0,7 \\
\mathrm{R}=x_{5} & 0,6 & 0 & 0 & 0 & 0 & 0,9 & 0,3 & 0 & 0 \\
x_{6} & 0 & 0 & 0 & 0 & 0,6 & 0 & 0,4 & 0 & 0 \\
x_{7} & 0 & 0 & 0 & 0 & 0,3 & 0,4 & 0 & 0,4 & 0 \\
x_{8} & 0,6 & 0 & 0 & 0 & 0 & 0 & 0,4 & 0 & 0,7 \\
x_{9} & 0 & 0 & 0,6 & 1 & 0 & 0 & 0 & 0,7 & 0
\end{array} \mid
$$

We raise the contiguity matrix to $2,3, \ldots, 9$ powers. Uniting them, we find an accessible matrix

$$
\begin{array}{c|ccccccccr}
\multicolumn{1}{c}{} & x_{1} & x_{2} & x_{3} & x_{4} & x_{5} & x_{6} & x_{7} & x_{8} & x_{9} \\
x_{1} & 1 & 1 & 0,7 & 0,7 & 0,7 & 0,7 & 0,4 & 0,7 & 0,7 \\
x_{2} & 0,7 & 1 & 0,6 & 0,7 & 0,7 & 0,7 & 0,4 & 0,7 & 0,7 \\
x_{3} & 0,6 & 0,6 & 1 & 0,7 & 0,6 & 0,6 & 0,4 & 0,7 & 0,7 \\
x_{4} & 0,6 & 0,6 & 0,6 & 1 & 0,6 & 0,6 & 0,4 & 0,7 & 0,7 \\
\mathrm{~N}=x_{5} & 0,6 & 0,6 & 0,6 & 0,6 & 1 & 0,9 & 0,4 & 0,6 & 0,6 \\
x_{6} & 0,6 & 0,6 & 0,6 & 0,6 & 0,6 & 1 & 0,4 & 0,6 & 0,6 \\
x_{7} & 0,4 & 0,4 & 0,4 & 0,4 & 0,4 & 0,4 & 1 & 0,4 & 0,4 \\
x_{8} & 0,6 & 0,6 & 0,6 & 1 & 0,6 & 0,6 & 0,4 & 1 & 1 \\
x_{9} & 0,6 & 0,6 & 0,6 & 1 & 0,6 & 0,6 & 0,4 & 0,7 & 1
\end{array}
$$

The corresponding expression (5) for this graph has the following form

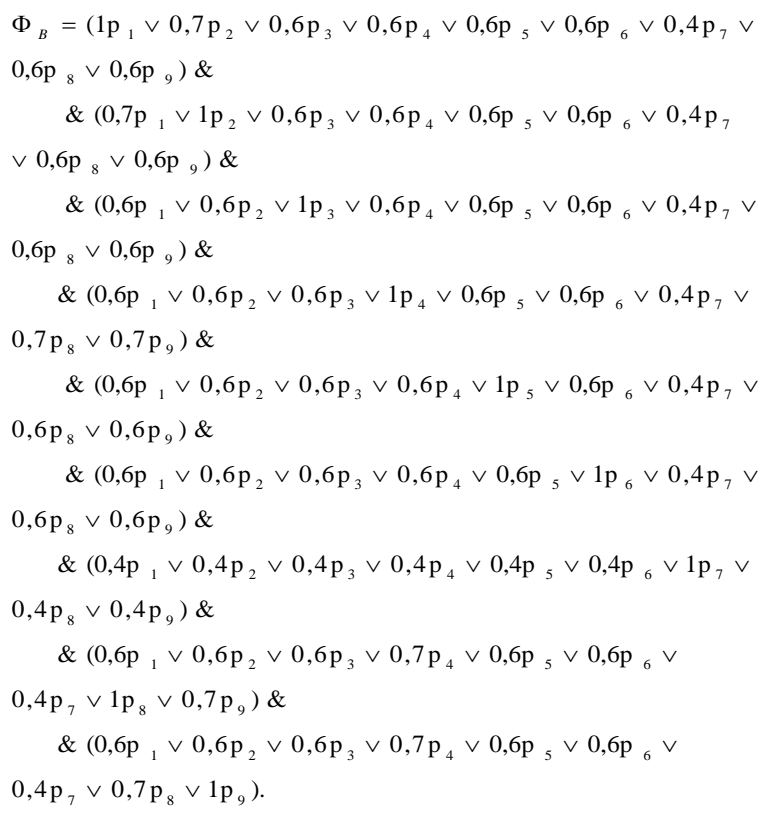

Before the first iteration of the algorithm vectors $\overline{V_{1}}$ and $\overline{V_{2}}$ are the following:

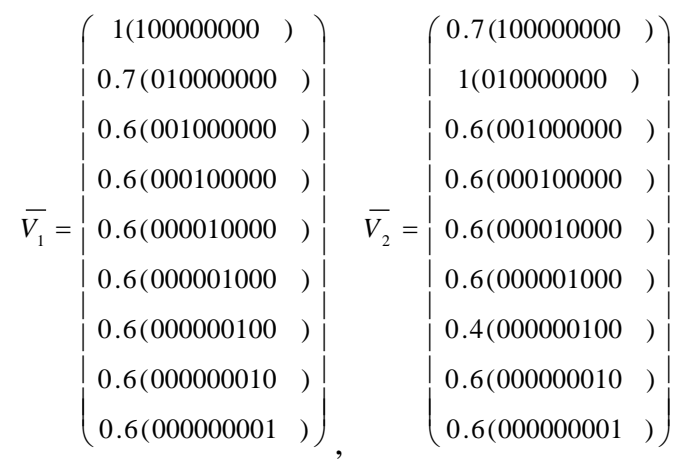

After the first iteration of the algorithm we have:

${\overline{V_{1}}}^{T}=(0.7(1000000000), 0.7(010000000)$,

0.6(001000000), 0.6(000100000), 0,6(000010000),

0.6(000001000), 0.4(000000100), 0.6(000000010), $0.6(000000001), 1(110000000))$.

After completing the iterations for $j=3,4, \ldots, 9$, finally we have:

${\overline{V_{1}}}^{T}=(0.4(1000000000), 0.4(010000000)$,

0.4(001000000), 0.4(000100000), 0,4(000010000), 0.4(000001000), 0.4(000000100), 0.4(000000010), 0.4(000000001), 0.6(100000100), 0.6(010000100), $0.6(001000100), 0.6(000100100), 0.6(000010100)$, 0.6(000001100), 0.6(000000110), 0.6(000000011), 0.7(101111100), 0.7(100011110), 0.7(101011101), 0.7(011111100), 0.7(011011110), 0.7(011011101), 1(11111111)).

So, the formula (4) for this graph has the form: 


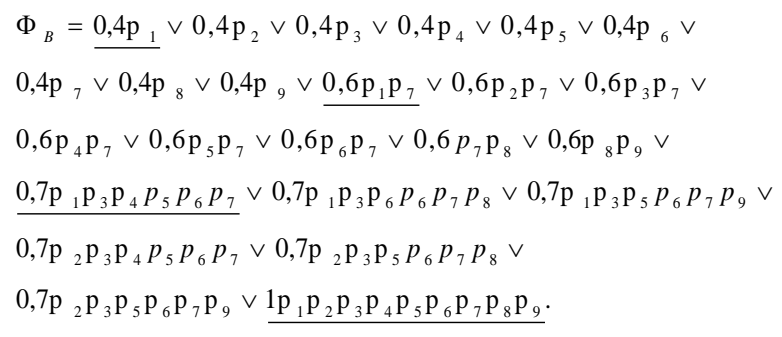

Graph $\tilde{G}$ has 24 subsets of vertices with the greatest vitality degree, which follows from the last equality, and the fuzzy set of vitality is defined as:

$$
V(\tilde{G})=\{\langle 0,4 / 1\rangle,\langle 0,6 / 2\rangle,\langle 0,7 / 6\rangle,\langle 1 / 9\rangle\} .
$$

The fuzzy set of vitality defines the following optimum allocation of the service centres: if we have 9 service centres, we should place them into all vertices. The greatest vitality degree equals to 1 . If we have 6 service centres, we should place them, for example, into vertices 1, 3, 4, 5, 6 and 7 (Inskaya, Barnaul, Novokuzneck, Yrga-2, Surzhenka, Kemerovo). The greatest vitality degree equals to 0.7 in this case. If we have 2 service centres, we should place the centres, for example, into vertices 1 and 7 (Inskaya, Kemerovo). In this case the degree of service equals to 0.6. If we have only one service centre then we can place it in any vertex (for example, Inskaya). Here, the degree of service equals to 0.4 . The fuzzy set of vitality also indicates that, for example, there is no need to place 3,4 , or 5 centers. In this case the greatest vitality degree will be the same as in the case of 2 centers.

\section{Conclusion}

The task of defining of the optimum allocation of centers was considered as the task of the definition of vitality fuzzy set of fuzzy graphs. It must be noted; that the introduced method makes it possible to define the best service allocations only if the centers are placed in the vertices of a graph (the case of generating new vertices on the edges is not considered). In our future work we are going to examine the problem of the centers' allocation in the temporal fuzzy graphs, i.e. the graphs, edges' membership functions of which change in discrete time.

\section{Acknowledgements}

This work has been supported by the Russian Foundation for Basic Research, Projects № 15-01-00149a, № 15-07-00185a.

\section{References}

[1] K. Clarke, Analytical and Computer Cartography, Englewood Cliffs, N.J.: Prentice Hall, 1995.

[2] P. Longley, M. Goodchild, D. Maguire and D. Rhind, Geographic Information Systems and Science, John Wiley \& Sons, Inc., New York, 2001.

[3] J. Zhang and M. Goodchild, Uncertainty in Geographical Information, Taylor \& Francis, Inc., New York, 2002.
[4] M. Goodchild, Modelling Error in Objects and Fields, in Accuracy of Spatial Databases, eds. M. Goodchild and S. Gopal, Taylor \& Francis, Inc., Basingstoke, 107--113, 1989.

[5] S. Belyakov, A. Bozhenyuk, M. Belykova, I. Rozenberg, Model Of Intellectual Visualization Of Geoinformation Service, In the 28th European Conference on Modelling and Simulation ECMS 2014, Brescia, 326--332, 2014.

[6] A. Kaufmann, Introduction a la combinatorique en vue des applications, Dunod, Paris 1968.

[7] N. Christofides, Graph Theory. An Algorithmic Approach, Academic press, London, 1976.

[8] J. Malczewski, GIS and multicriteria decision analysis, John Willey and Sons, New York, 1999.

[9] L. Bershtein and A. Bozhenuk, Fuzzy graphs and fuzzy hypergraphs, In J. Dopico, J. de la Calle, and A. Sierra, editors, Encyclopedia of Artificial Intelligence Information SCI, pages 704-709, Hershey, New York, 2008.

[10] L. Bershtein and A. Bozhenuk, Maghout method for determination of fuzzy independent, dominating vertex sets and fuzzy graph kernels, $J$. General Systems, 30: 45--52, 2001.

[11] A. Bozhenyuk and I. Rozenberg, Allocation of Service Centers in the GIS with the Largest Vitality Degree, In S. Greco et al., editors, IPMU 2012, Part II, Communications in Computer and Information Science, CCIS 298, Springer-Verlag, Berlin Heidelberg, 98--106, 2012.

[12] A. Bozhenyuk, I. Rozenberg, T. Starostina. A Method of Increase of Vitality Degree of Fuzzy Graph // Proceedings of 13th Zittau Fuzzy Colloquium. September 13-15. Hochschule Zittau/Goerlitz, Zittau pp. 235--240. 2006.

[13] L. Bershtein, A. Bozhenyuk, I. Rozenberg. Optimum allocation of centers in transportation networks by means of fuzzy graph bases // Proceedings of the 8th conference of the European Society for Fuzzy Logic and Technology (EUSFLAT-13). Advances in Intelligent Systems Research. Editors: Gabriella Pasi, Javier Montero, Davide Ciucci, 11 13 September 2013. Atlantis Press, pp.230--235, 2013.

[14] L. Bershtein, A. Bozhenyuk, I. Rozenberg. Service Centers Finding by Fuzzy Antibases of Fuzzy Graph // Proceedings of the International Workshop on Soft Computing Applications and Knowledge Discovery (SCAKD 2011). Moscow, Russia, June 25, 12--22, 2011.

[15] I. Rozenberg, C. Gittis and D. Svyatov, Geoinformation System Object Land, in Proc. IPI RAN "Systems and Means of Informatics", Science, Moscow, 2000. 